\title{
TIME VARIATION OF KEPLER TRANSITS INDUCED BY STELLAR ROTATING SPOTS-A WAY TO DISTINGUISH BETWEEN PROGRADE AND RETROGRADE MOTION. I. THEORY
}

\author{
Tsevi MazeH ${ }^{1,2}$, Tomer Holczer ${ }^{1}$, And Avi ShPORER ${ }^{3,4,5}$ \\ ${ }^{1}$ School of Physics and Astronomy, Raymond and Beverly Sackler Faculty of Exact Sciences, \\ Tel Aviv University, Tel Aviv 69978, Israel; mazeh@post.tau.ac.il \\ 2 The Jesus Serra Foundation Guest Program, Instituto de Astrofsica de Canarias, C. via Lactea S/N, E-38205 La Laguna, Tenerife, Spain \\ ${ }^{3}$ Division of Geological and Planetary Sciences, California Institute of Technology, Pasadena, CA 91125, USA \\ ${ }^{4}$ Jet Propulsion Laboratory, California Institute of Technology, 4800 Oak Grove Drive, Pasadena, CA 91109, USA \\ Received 2014 June 23; accepted 2014 December 30; published 2015 February 20
}

\begin{abstract}
Some transiting planets discovered by the Kepler mission display transit timing variations (TTVs) induced by stellar spots that rotate on the visible hemisphere of their parent stars. An induced TTV can be observed when a planet crosses a spot and modifies the shape of the transit light curve, even if the time resolution of the data does not allow the detection of the crossing event itself. We present an approach that can, in some cases, use the derived TTVs of a planet to distinguish between a prograde and a retrograde planetary motion with respect to the stellar rotation. Assuming a single spot darker than the stellar disk, spot crossing by the planet can induce measured positive (negative) TTV, if the crossing occurs in the first (second) half of the transit. On the other hand, the motion of the spot toward (away from) the center of the stellar visible disk causes the stellar brightness to decrease (increase). Therefore, for a planet with prograde motion, the induced TTV is positive when the local slope of the stellar flux at the time of transit is negative, and vice versa. Thus, we can expect to observe a negative (positive) correlation between the TTVs and the photometric slopes for prograde (retrograde) motion. Using a simplistic analytical approximation, and also the publicly available SOAP-T tool to produce light curves of transits with spotcrossing events, we show for some cases how the induced TTVs depend on the local stellar photometric slopes at the transit timings. Detecting this correlation in Kepler transiting systems with high enough signal-to-noise ratio can allow us to distinguish between prograde and retrograde planetary motions. In upcoming papers we present analyses of the KOIs and Kepler eclipsing binaries, following the formalism developed here.
\end{abstract}

Key words: planetary systems - starspots - stars: rotation - techniques: photometric

\section{INTRODUCTION}

Formation and evolutionary processes of stellar and planetary systems are expected to leave their imprint on the present-day systems. One such imprint is the stellar obliquity, the angle between the stellar spin axis and the orbital angular momentum axis, also referred to as the spin-orbit angle. For star-planet systems the measurement of this angle is a matter of intense study in recent years (e.g., Triaud et al. 2010; Moutou et al. 2011; Winn et al. 2011; Albrecht et al. 2012), primarily for hot Jupiters-gas-giant planets at short-period orbits. Some of the systems were found to be aligned, in a prograde orbit with the spin-orbit angle close to zero, while others were found to be misaligned, including systems in retrograde motion where the spin-orbit angle is close to $180^{\circ}$ (e.g., Hébrard et al. 2011; Winn et al. 2011).

The growing sample and the wide range of spin-orbit angles measured for hot Jupiters can be used for studying their orbital evolutionary history. For example, Winn et al. (2010) have noticed that hot stars, with an effective temperature above $6250 \mathrm{~K}$, tend to have a wide obliquity range, while cool stars tend to have low obliquities, mostly consistent with wellaligned orbits. This was confirmed by a study of a larger sample by Albrecht et al. (2012) and is consistent with the results of Schlaufman (2010) and Hansen (2012) who used different approaches. Those authors suggested that some mechanisms can cause the planetary orbit to attain large obliquity (e.g., Fabrycky \& Tremaine 2007; Naoz et al. 2011;

\footnotetext{
${ }^{5}$ Sagan Fellow.
}

Batygin 2012). Then, tidal interaction with the host star (e.g., Winn et al. 2010) or magnetic braking (e.g., Dawson 2014) acts to realign the orbit. Since these processes are probably inefficient for hot stars, those systems might still retain their wide obliquity range.

So far spin-orbit alignment has been studied primarily through the Rossiter-McLaughlin (RM) effect (Holt 1893; Schlesinger 1910; Rossiter 1924; McLaughlin 1924), originally suggested for stellar eclipsing binaries, and observed by monitoring the anomalous radial-velocity signal during eclipse, as the eclipsing star moves across the disk of the eclipsed star. The RM effect is sensitive to the sky-projected component of the spin-orbit angle, and was successfully measured for many transiting planet systems (e.g., Queloz et al. 2000; Winn et al. 2006; Triaud et al. 2010), transiting brown dwarfs, and low-mass star systems (Triaud et al. 2013), and stellar binaries (Albrecht et al. 2007, 2009, 2011, 2014).

The line-of-sight component of the spin-orbit angle can be measured using asteroseismology (Gizon \& Solanki 2003; Chaplin et al. 2013), or the observed rotational broadening of spectral lines, if the host star radius and rotation period are known with sufficient precision (Hirano et al. 2012, 2014; see also Schlaufman 2010). However, these two methods require obtaining new data for each target, using valuable resources (e.g., large telescopes or Kepler short-cadence data). Other methods have been presented, based on stellar gravitational darkening (Barnes 2009; Barnes et al. 2011; Szabo 2011) and the beaming effect (Photometric RM-Groot 2012; Shporer et al. 2012). 
An interesting approach was taken by Nutzman et al. (2011) and Sanchis-Ojeda et al. (2011), who use the brief photometric signals during transit induced by the transiting object moving across spots located on the surface of the host object. This is based on the fact that many stars show photometric modulations resulting from the combination of stellar rotation and nonuniform longitudinal spots distribution (e.g., Irwin et al. 2009; Hartman et al. 2011; McQuillan et al. 2014). When such a star displays transits by an orbiting planet, the transiting object might momentarily eclipse a stellar spot, inducing an increase in observed flux, if the surface brightness of the spot-covered area is lower than that of the non-spotted areas. The derivation of the stellar obliquity requires identification of such "spotcrossing" events within a few transits, and estimate the spot and the planet phases within their motion over the stellar disk. The method has since been applied to additional systems using high-speed Kepler and CoRoT data (Deming et al. 2011; Désert et al. 2011; Sanchis-Ojeda \& Winn 2011; Sanchis-Ojeda et al. 2012, 2013).

We present here another version of this approach that does not require such high-speed photometry. Instead, we use the fact that a spot-crossing event can induce measurable transit time variation (TTV; e.g., Sanchis-Ojeda et al. 2011; Fabrycky et al. 2012; Mazeh et al. 2013; Szabó et al. 2013; Oshagh et al. 2013b), even for data that cannot resolve the event itself. Our approach relies on the expected correlation between the induced TTV and the corresponding local photometric slope immediately outside the transit, presumably induced by the same spot. Detected correlation or anti-correlation between the TTVs and their local slope can in principle differentiate between prograde and retrograde rotation of the primary star in stellar binaries or star-planet systems.

We present here the basic concept and develop an analytical simplistic approximation for the induced TTVs and the photometric slope. We also use the work of Boisse et al. (2012) and Oshagh et al. (2013a), who developed a numerical tool, SOAP-T ${ }^{6}$, to simulate a planetary transit light curve which includes a spot-crossing event. Oshagh et al. (2013b) used SOAP-T to derive detailed transit light curves, and then fitted them with transit templates to obtain the expected TTVs, very similar to what is performed when deriving the TTVs from the Kepler actual data (e.g., Mazeh et al. 2013). We show that our approximation yields TTVs with the same order of magnitude as the results of Oshagh et al. (2013b). Using our approximation and the SOAP-T tool we show that in some cases we can expect a negative (positive) correlation between the TTVs induced by spot crossing and the local photometric slopes at the transit timings for prograde (retrograde) motion of the planet. We also discuss the limitations of this approach when applied to real data, showing that it can be applied only to a limited number of systems.

The paper is organized as follows. Section 2 outlines the basics of our approach, while Section 3 presents the analytical approximation for the induced TTV for different cases, and Section 4 compares our approximation with numerical simulations we performed and those of Oshagh et al. (2013b). In Section 5 we derive the expected derivative of the stellar brightness at the time of transit, and in Section 6 display the expected correlation between the induced TTVs and

\footnotetext{
http://www.astro.up.pt/resources/soap-t/
}

the stellar photometric slopes. Finally, Section 7 discusses our results and the severe limitations of its applicability to real data.

The present paper is the first of three studies. The next study (T. Holczer et al. 2015, in preparation) presents our analysis for the Kepler planet candidates (Batalha et al. 2013). In that paper we show that indeed a few systems do show highly significant correlation between their derived TTVs and the local photometric derivatives, as predicted by this work. A forthcoming paper will present our analysis of the Kepler eclipsing stellar binaries (Slawson et al. 2011).

\section{THE PRINCIPLE OF THE APPROACH}

To present our approach, we consider a transiting planet that crosses a stellar spot during its apparent motion over the stellar disk. Let us assume, for the sake of simplicity, that only one spot is present on the stellar disk and that the stellar rotation and orbital axes are parallel to each other. This includes both prograde (complete alignment, with obliquity of $0^{\circ}$ ) and retrograde (obliquity of $180^{\circ}$ ) configurations. The sign of the induced time shift depends on whether the spot-crossing event occurs in the first (positive TTV) or second (negative TTV) half of the transit, which is determined by the location of the spot on the stellar disk at the time of transit.

As depicted in Figures 1 and 2, the location of the spot over the stellar disk determines whether the star is becoming brighter or dimmer at the time of transit. When the spot is moving toward (away from) the center of the disk the stellar intensity is decreasing (increasing), because of the aspect effect, which changes the effective area of the spot on the stellar visible disk - the projected area of the spot onto the sky plane. Therefore, when the spot is on the disk edge its effective area is minimal. On the other hand, the effective area reaches its maximum when the spot is at its closest position to the center of the visible disk, when the stellar surface is (almost) perpendicular to our line of sight. Another phenomenon, which also causes the star to become fainter when the spot moves toward the center of the disk is the limb-darkening effect, which is ignored at this point of the discussion.

Now, when the stellar rotation and planetary motion have the same sense of rotation, the spot-crossing event in the first (second) half of the transit should always occur when the spot is moving toward (away from) the center of the disk. Therefore the signs of the induced TTV and the slope of the stellar brightness at the time of transit should be opposite. This is depicted in Figure 1 for prograde motion. For retrograde motion, positive TTV should be associated with positive slope, as depicted in Figure 2.

Therefore, we expect negative correlation between the derived TTVs and the corresponding stellar photometric slopes for a system with planetary prograde motion and positive correlation for a system with retrograde motion. In the next sections we will show that this is indeed the case for a limited number of cases by deriving analytical approximations for the TTVs and the photometric derivatives and by numerical simulations for the TTVs.

\section{ANALYTICAL APPROXIMATION FOR THE TTV INDUCED BY THE SPOT-CROSSING EVENT}

\subsection{Center-of-light Approximation}

To present the concept behind our method in a more quantitative way, Figure 3 shows a simplified schematic 

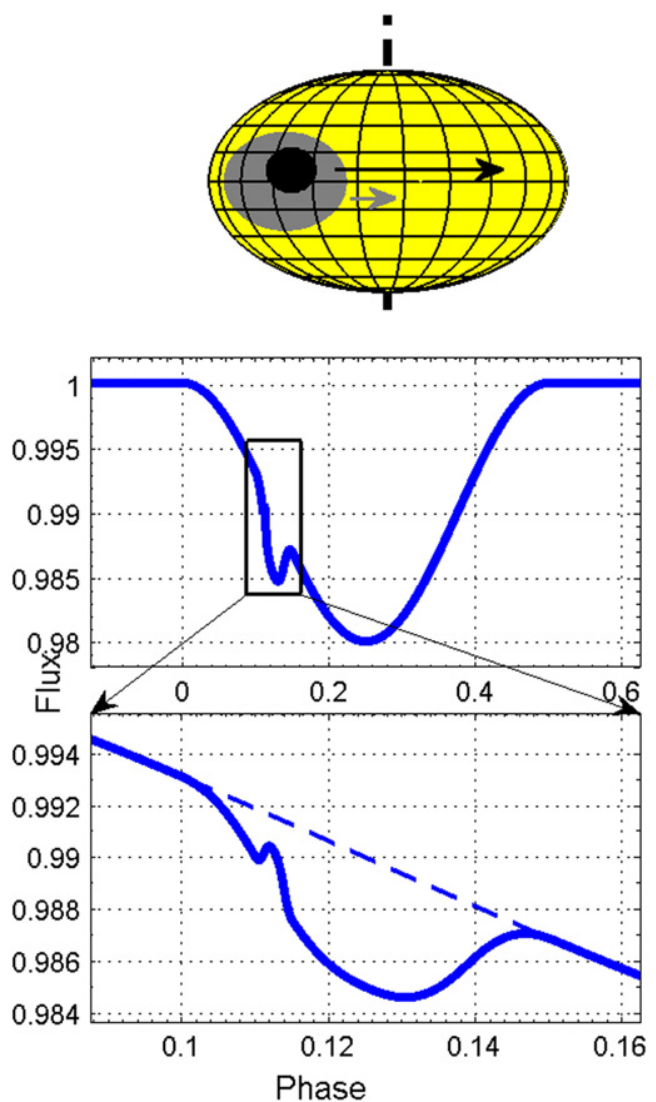
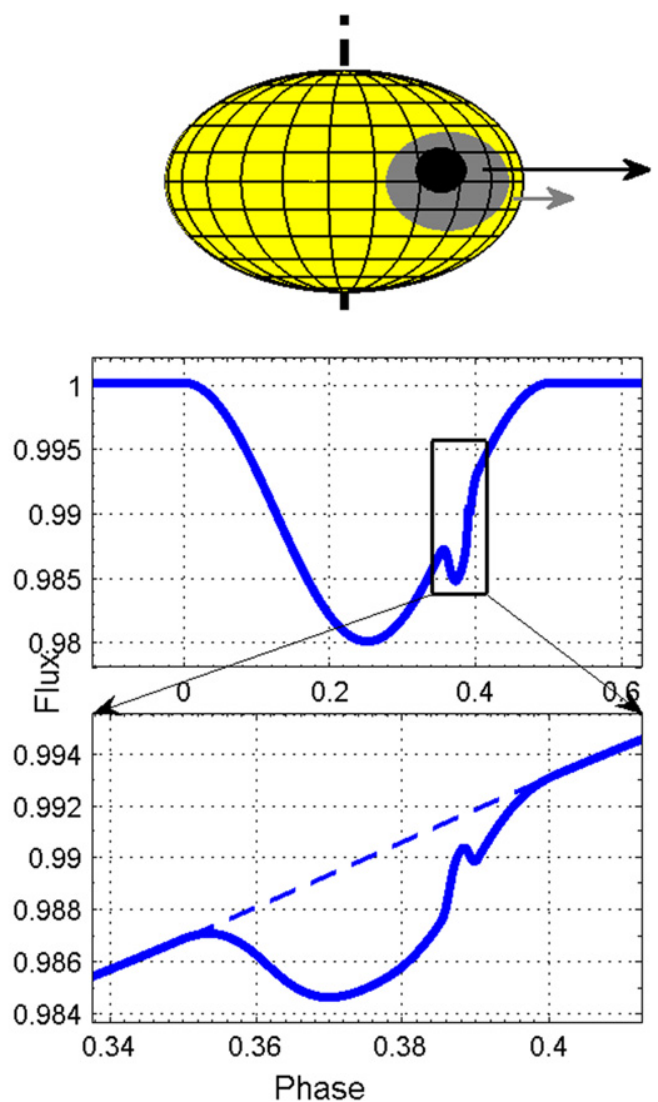

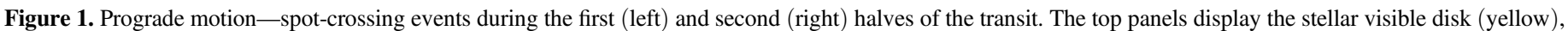

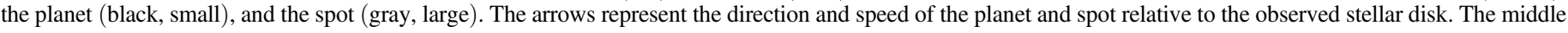

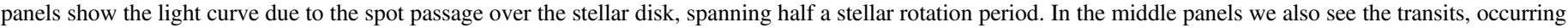

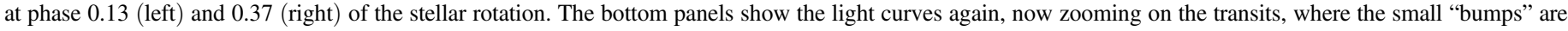

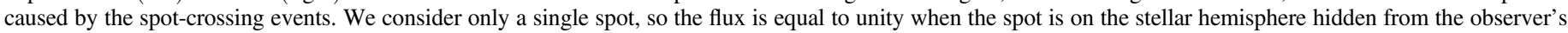

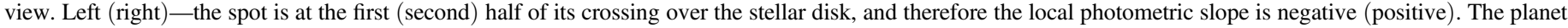
is at the first (second) half of the transit and therefore the derived transit timing shift (while the spot-crossing is unresolved) is positive (negative).

diagram of a transit light curve with a single spot-crossing event. We neglect the transit ingress and egress finite duration of both the transit and the spot-crossing event. We also assume that at the time of each transit there is only one circular spot on the stellar disk. In the figure we also neglect the limb-darkening photometric modulation, and will consider this effect later.

In the figure, $\delta_{\mathrm{tr}}$ and $\delta_{\mathrm{sc}}$ are the depth of the transit and the amplitude of the photometric increase inside the transit due to the spot-crossing event, respectively, $\Delta_{\text {tr }}$ and $\Delta_{\mathrm{sc}}$ are the duration of the transit and the spot-crossing event, respectively, and $t_{s c}$ is the timing of the spot-crossing event relative to midtransit time.

From the figure one can see, using "center-of-light" formulation, that we expect the TTV induced by the spotcrossing event to be:

$$
\mathrm{TTV}_{\mathrm{sc}} \simeq-t_{\mathrm{sc}} \frac{\delta_{\mathrm{sc}} \Delta_{\mathrm{sc}}}{\delta_{\mathrm{tr}} \Delta_{\mathrm{tr}}-\delta_{\mathrm{sc}} \Delta_{\mathrm{sc}}} .
$$

This result is similar to Equation (3) of Sanchis-Ojeda et al. (2011) after neglecting $\delta_{\mathrm{sc}} \Delta_{\mathrm{sc}}$ in the denominator. We will adopt this approximation below.

\subsection{Basic Model}

To derive the analytical simplistic model we first consider a case for which
1. the impact parameter of the spot and the planet are both equal to zero, namely, that they both cross the center of the stellar disk, and

2. there is no limb darkening.

We lessen these two assumptions below.

We denote the location of the spot on the stellar disk by the angle $\psi$, which is the angle between the observer and the spot, as seen from the stellar center. If the motion of the spot is equatorial, then $\psi$ is the longitude of the spot on the stellar visible hemisphere:

$$
\psi(t)=\omega_{*} t
$$

where $\omega_{*}$ is the stellar angular velocity. When the spot is on the stellar limb entering the visible hemisphere, $\psi$ gets the value of $-\pi / 2$, and when the location of the spot is in the middle of its visible chord $\psi=0$.

We denote the angle corresponding to the spot crossing by $\psi_{\mathrm{sc}}$. The sky-projected distance of the spot from the stellar center, as seen by the observer, is $d_{\mathrm{sc}}=R_{*} \sin \psi_{\mathrm{sc}}$, where $R_{*}$ is the stellar radius. The timing of the spot-crossing event, measured relative to the middle of the transit, is therefore

$$
t_{\mathrm{sc}}=\frac{\Delta_{\mathrm{tr}}}{2} \sin \psi_{\mathrm{sc}}
$$



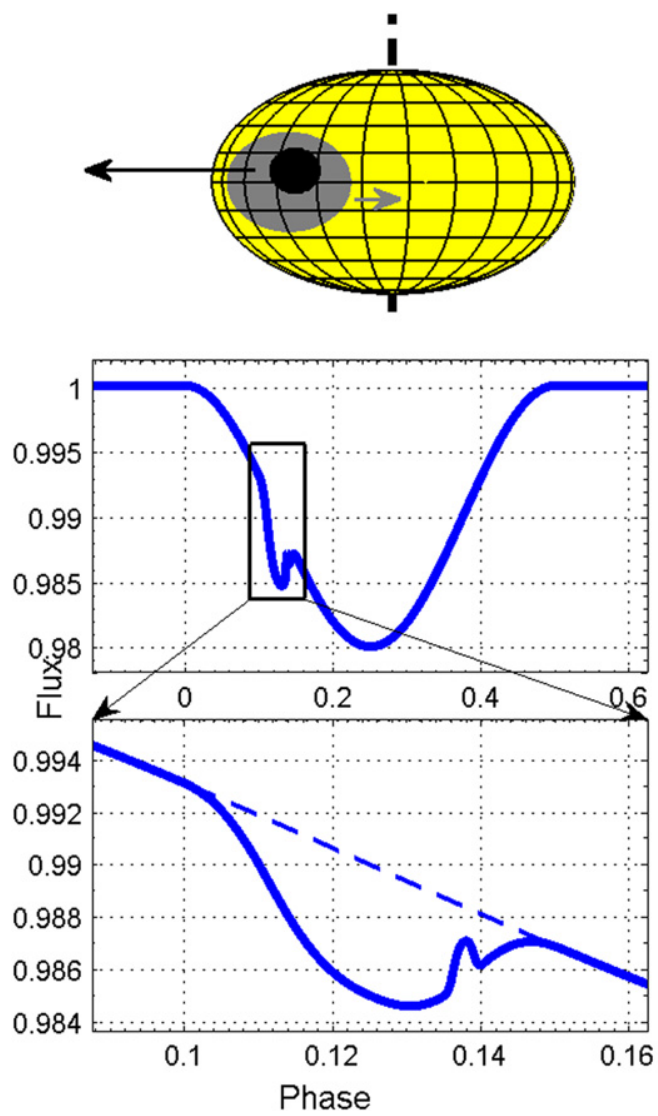
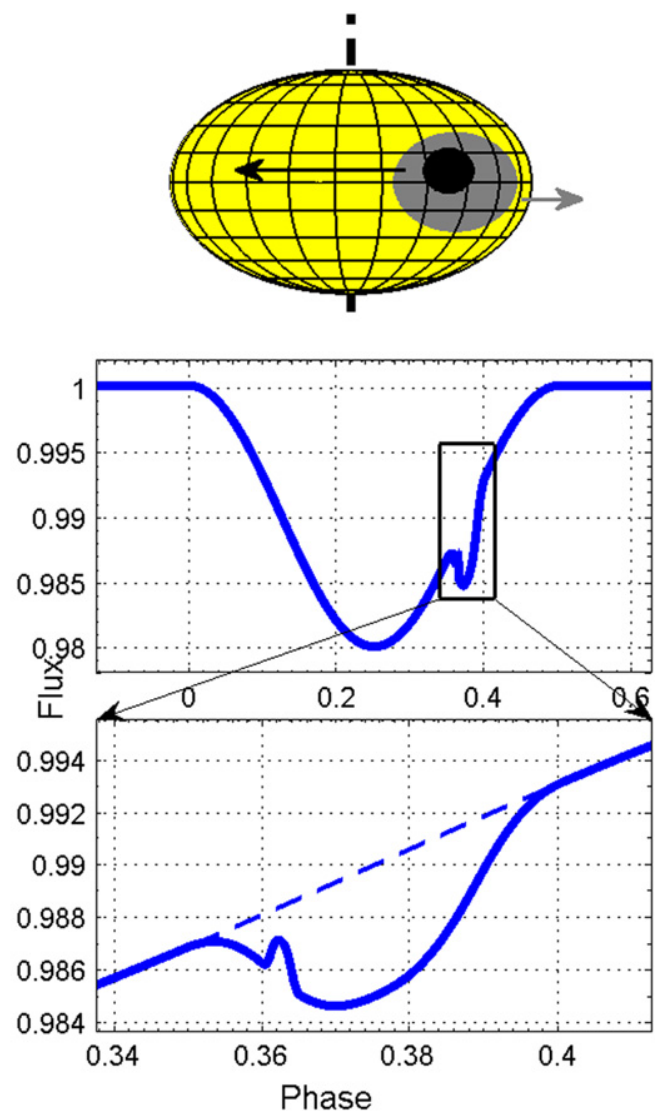

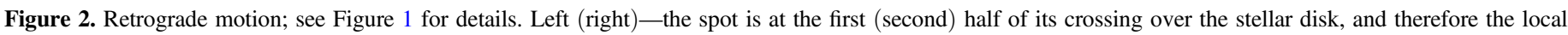

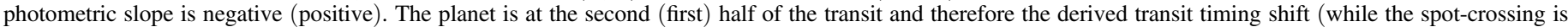
unresolved) is negative (positive).

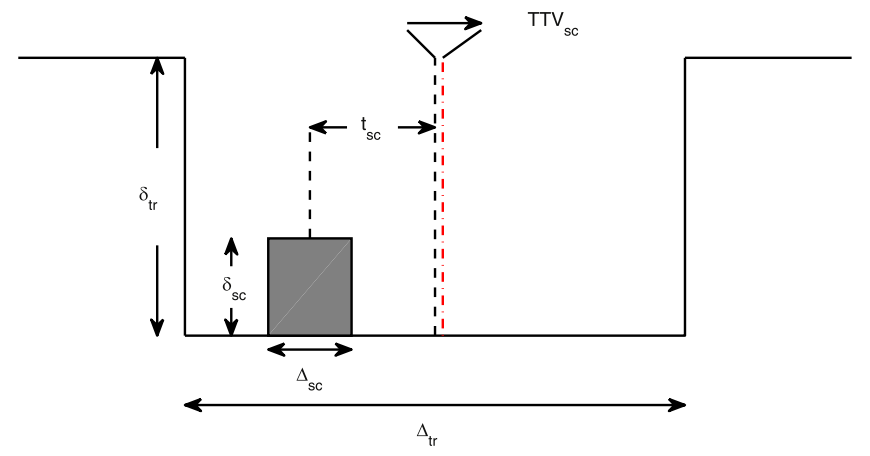

Figure 3. Schematic diagram of a transit light curve with a spot-crossing event The transit depth is $\delta_{\mathrm{tr}}$, while $\delta_{\mathrm{sc}}$ is the flux increase due to the spot-crossing event, $\Delta_{\mathrm{tr}}$ and $\Delta_{\mathrm{sc}}$ are the transit and spot-crossing durations, and $t_{s c}$ is the timing of the spot-crossing event relative to the mid transit. The vertical dashed black line represents the expected mid-transit timing, without any spot-crossing event, while the red dashed-dotted line represents the new mid-transit measurement, due to the shift induced by the spot crossing. The difference between the two lines, $T T V_{s c}$, is the induced TTV. Approximately, $\mathrm{TTV}_{\mathrm{sc}} \simeq-t_{\mathrm{sc}} \times \delta_{\mathrm{sc}} \Delta_{\mathrm{sc}} /\left(\delta_{\mathrm{tr}} \Delta_{\mathrm{tr}}-\delta_{\mathrm{sc}} \Delta_{\mathrm{sc}}\right)$.

In order to estimate the induced TTV, let us consider two extreme cases: a small spot, for which

$$
R_{\text {spot }} \ll R_{\mathrm{pl}} \ll R_{*},
$$

and a large spot, for which

$$
R_{\mathrm{pl}} \ll R_{\text {spot }} \ll R_{*},
$$

where $R_{\mathrm{pl}}$ and $R_{\mathrm{spot}}$ are the radii of the planet and the spot, respectively.
For both cases we introduce a darkness parameter, $0<\alpha<1$, which measures the surface brightness of the spot relative to the surface brightness of the star immediately outside the spot. A completely dark spot would have $\alpha=0$, while $\alpha$ close to unity means the spot is almost as bright as the unspotted stellar area.

For the small-spot approximation we can assume that the spot is completely covered by the planet during the spotcrossing event and therefore

$$
\text { Small spot: } \quad \begin{aligned}
\delta_{\mathrm{sc}} & \simeq(1-\alpha)\left(\frac{R_{\mathrm{spot}}}{R_{*}}\right)^{2} \cos \psi_{\mathrm{sc}}, \\
\Delta_{\mathrm{sc}} & \simeq \Delta_{\mathrm{tr}}\left(\frac{R_{\mathrm{pl}}}{R_{*}}\right) .
\end{aligned}
$$

As noted above, the factor $\cos \psi_{\mathrm{sc}}$ comes from the fact that the effective area of the spot is reduced by the aspect ratio, which is a function of the spot position on the visible stellar disk.

For the large-spot approximation the planet is fully contained in the spotted area during the spot-crossing event, and therefore we get

$$
\text { Large spot: } \begin{aligned}
\delta_{\mathrm{sc}} & \simeq(1-\alpha)\left(\frac{R_{\mathrm{pl}}}{R_{*}}\right)^{2}, \\
\Delta_{\mathrm{sc}} & \simeq \Delta_{\mathrm{tr}}\left(\frac{R_{\mathrm{spot}}}{R_{*}}\right) \cos \psi_{\mathrm{sc} .}
\end{aligned}
$$

Here the factor $\cos \psi_{\mathrm{sc}}$ comes from the fact that the time to 
cross the spot by the relatively small planet is reduced by the same aspect ratio.

We now approximate the TTV to be

$$
\mathrm{TTV}_{\mathrm{sc}} \simeq-t_{\mathrm{sc}} \frac{\delta_{\mathrm{sc}}}{\delta_{\mathrm{tr}}} \frac{\Delta_{\mathrm{sc}}}{\Delta_{\mathrm{tr}}}
$$

and the transit depth $\delta_{\text {tr }}$ to be on the order of $\left(R_{\mathrm{pl}} / R_{*}\right)^{2}$. We therefore get for the small-spot approximation

$$
\text { small spot: } \begin{aligned}
\mathrm{TTV}_{\mathrm{sc}} & \simeq-t_{\mathrm{sc}}(1-\alpha)\left(\frac{R_{\mathrm{spot}}}{R_{*}}\right)^{2} \frac{R_{\mathrm{pl}}}{R_{*}}\left(\frac{R_{\mathrm{pl}}}{R_{*}}\right)^{-2} \cos \psi_{\mathrm{sc}} \\
& =-t_{\mathrm{sc}}(1-\alpha) \frac{R_{\mathrm{spot}}^{2}}{R_{\mathrm{pl}} R_{*}} \cos \psi_{\mathrm{sc}}
\end{aligned}
$$

and for the large-spot approximation

$$
\text { large spot: } \operatorname{TTV}_{\mathrm{sc}} \simeq-t_{\mathrm{sc}}(1-\alpha) \frac{R_{\mathrm{spot}}}{R_{*}} \cos \psi_{\mathrm{sc}} .
$$

Note that when $R_{\text {spot }} \rightarrow R_{\mathrm{pl}}$, Equation (9) $\rightarrow$ Equation (10). To ease the discussion we define $\mathcal{R}$ as:

$$
\mathcal{R}= \begin{cases}\frac{R_{\mathrm{spot}}^{2}}{R_{\mathrm{pl}} R_{*}} & \text { for small spot } \\ \frac{R_{\mathrm{spot}}}{R_{*}} & \text { for large spot. }\end{cases}
$$

Using Equation (3) we get:

$$
\mathrm{TTV}_{\mathrm{sc}} \simeq-(1-\alpha) \mathcal{R} \frac{\Delta_{\mathrm{tr}}}{2} \cos \psi_{\mathrm{sc}} \sin \psi_{\mathrm{sc}},
$$

which is valid both for the small- and large-spot approximations. The maximum observed TTV induced by the spot crossing is

$$
\max \left\{\operatorname{TTV}_{\mathrm{sc}}\right\} \simeq \frac{(1-\alpha)}{4} \mathcal{R} \Delta_{\mathrm{tr}}
$$

\subsection{Models for Limb Darkening, Impact Parameter, and Obliquity}

\subsubsection{Limb Darkening}

To include the stellar limb darkening effect in our model, we consider a quadratic limb-darkening law of $S=1-g_{1}(1-\cos \psi)-g_{2}(1-\cos \psi)^{2}$, where $S$ is the scaled stellar surface brightness and $g_{1}$ and $g_{2}$ are the two limbdarkening coefficients, such that $g_{1}+g_{2}<1$.

The induced TTV is proportional to $\delta_{\mathrm{sc}}$, the increase of the stellar brightness during the spot crossing, which depends linearly on the stellar surface brightness $S$, which is now a function of $\psi$. Therefore we get

$$
\begin{aligned}
\operatorname{TTV}_{\mathrm{sc}}= & -(1-\alpha) \mathcal{R} \frac{\Delta_{\mathrm{tr}}}{2} \cos \psi(t) \sin \psi(t) \\
& \times\left\{1-g_{1}(1-\cos \psi)-g_{2}(1-\cos \psi)^{2}\right\} \\
= & -(1-\alpha) \mathcal{R} \frac{\Delta_{\text {tr }}}{2}\left\{\left(1-g_{1}-g_{2}\right) \sin \psi(t)\right. \\
& +\left(g_{1}+2 g_{2}\right) \sin \psi(t) \cos \psi(t) \\
& \left.-g_{2} \sin \psi(t) \cos ^{2} \psi(t)\right\} \cos \psi(t) .
\end{aligned}
$$

Note that because of the limb darkening the transit light curve does not have a rectangular shape, so our Equation (1) should be modified. Nevertheless, as this analytical approach is aimed only to understand the features of the TTVs as a function of the spot-crossing phase, we neglect this effect that will affect all phases alike.

\subsubsection{Impact Parameter}

Another extension of our simplistic model accounts for a non-zero impact parameter, $b=\cos \theta$. Note that the stellar rotation is, as before, orthogonal to our line of sight. In this extension of the simplistic model, both planet and spot still have the same impact parameter, namely, both move along the same chord on the stellar disk, a chord that does not go through the center of the disk. Therefore, the spot moves at a colatitude $\theta_{\text {spot }}=\theta$, with an impact parameter $b_{\text {spot }}=\cos \theta_{\text {spot. }}$. In such a case, the angle $\psi$ fulfill the relation

$$
\cos \psi=\sin \theta \cos \phi,
$$

where now $\phi$ is the longitude of the planet, and $\phi=0$ is when the planet crosses the projection of the stellar rotational axis. The range of $\psi$ is now different: $\pi / 2-\theta \leqslant|\psi| \leqslant \pi / 2$, and the timing of the spot crossing is

$$
t_{\mathrm{sc}}=\frac{\Delta_{\mathrm{tr}}^{b}}{2} \sin \phi_{\mathrm{sc}},
$$

where $\Delta_{\mathrm{tr}}^{b}$ is the transit duration when $b \neq 0$. A good approximation would be $\Delta_{\mathrm{tr}}^{b}=\Delta_{\mathrm{tr}} \sin \theta$.

We now separate the discussion for the small and large spot approximations. For small spot, the duration of the spotcrossing event, $\Delta_{\mathrm{sc}}$, is still the same as for $b=0$, but the transit duration is shorter by a factor of $\sin \theta$. The flux increase depends on $\cos \psi$, as for $b=0$. We can therefore write

$$
\begin{aligned}
\text { Small spot: } \delta_{\mathrm{sc}} & \simeq(1-\alpha)\left(\frac{R_{\mathrm{spot}}}{R_{*}}\right)^{2} \sin \theta \cos \phi_{\mathrm{sc}}, \\
\Delta_{\mathrm{sc}} & \simeq \Delta_{\mathrm{tr}}^{b}\left(\frac{R_{\mathrm{pl}}}{R_{*}}\right) \frac{1}{\sin \theta} .
\end{aligned}
$$

Combining these expressions we get

$$
\text { small spot: } \quad \operatorname{TTV}_{\mathrm{sc}} \simeq-(1-\alpha) \mathcal{R} \frac{\Delta_{\mathrm{tr}}^{b}}{2} \cos \phi_{\mathrm{sc}} \sin \phi_{\mathrm{sc}} \text {. }
$$

For the large spot case, the duration of the spot-crossing event, $\Delta_{\mathrm{sc}}$, is now different, as the planet is crossing a spot that forms an ellipse on the stellar disk, whose axes are $R_{\text {spot }}$ and $R_{\text {spot }} \cos \psi$. One can show that the length of the planet's path 
on the spotted area is $R_{\text {spot }} \sqrt{\cos ^{2} \theta+\sin ^{2} \theta \cos ^{2} \phi_{\mathrm{sc}}}$. We therefore get

$$
\text { large spot: } \begin{aligned}
\delta_{\mathrm{sc}} & \simeq(1-\alpha)\left(\frac{R_{\mathrm{pl}}}{R_{*}}\right)^{2}, \\
\Delta_{\mathrm{sc}} & \simeq \Delta_{\mathrm{tr}}^{b}\left(\frac{R_{\mathrm{spot}}}{R_{*}}\right) \sqrt{\cot ^{2} \theta+\cos ^{2} \phi_{\mathrm{sc}}},
\end{aligned}
$$

and thus

$$
\begin{aligned}
\text { large spot: } \mathrm{TTV}_{\mathrm{sc}} \simeq & -(1-\alpha) \\
& \times \mathcal{R} \frac{\Delta_{\mathrm{tr}}^{b}}{2} \sqrt{\cot ^{2} \theta+\cos ^{2} \phi_{\mathrm{sc}}} \\
& \times \sin \phi_{\mathrm{sc}} .
\end{aligned}
$$

We can see that for a non-vanishing impact parameter there is a difference between the large and small planet cases, unlike in the basic model. The difference is due to the $\cot ^{2} \theta$ term under the square sign in Equation (20). Note that the approximation of the large spot is not valid for $|\phi| \simeq \pi / 2$, where the projected area of the spot is small. Hence, we inserted into the calculation of the large-spot case a correction factor that turns the TTV expression to be similar to the smallspot one when $|\phi| \rightarrow \pi / 2$. This was done by multiplying the $\cot ^{2} \theta$ term with a Fermi function that is approximately unity, except for $|\phi| \rightarrow \pi / 2$, when the correction factor goes to zero.

\subsubsection{Limb Darkening and Impact Parameter}

To further extend our simplistic model, we consider now a case for non-zero impact parameter and quadratic limb darkening together. As before, we divide the discussion between the cases of small and large spot. Following Equation (18), but now multiplying it by the limb darkening brightness factor, we get for the small spot case:

small spot: $\mathrm{TTV}_{\mathrm{sc}} \simeq-(1-\alpha) \mathcal{R} \frac{\Delta_{\text {tr }}^{b}}{2}\left\{\left(1-g_{1}-g_{2}\right) \sin \phi(t)\right.$

$$
\begin{aligned}
& +\left(g_{1}+2 g_{2}\right) \sin \theta \sin \phi(t) \cos \phi(t) \\
& \left.-g_{2} \sin ^{2} \theta \sin \phi(t) \cos ^{2} \phi(t)\right\} \\
& \times \cos \phi(t),
\end{aligned}
$$

while for the large spot case, following Equation (20), we get:

$$
\begin{aligned}
\text { large spot: } \mathrm{TTV}_{\mathrm{sc}} \simeq & -(1-\alpha) \mathcal{R} \frac{\Delta_{\mathrm{tr}}^{b}}{2}\left\{\left(1-g_{1}-g_{2}\right) \sin \phi(t)\right. \\
& +\left(g_{1}+2 g_{2}\right) \sin \theta \sin \phi(t) \cos \phi(t) \\
& \left.-g_{2} \sin ^{2} \theta \sin \phi(t) \cos ^{2} \phi(t)\right\} \\
& \times \sqrt{\cot ^{2} \theta+\cos ^{2} \phi(t)} .
\end{aligned}
$$

\subsubsection{Stellar Obliquity}

The last case we consider is when the apparent planetary chord along the stellar disk goes through the center $\left(b_{\mathrm{pl}}=0\right)$, but is inclined with the angle $\eta$ relative to the stellar equator. We nevertheless assume that in some transits spot-crossing events happen, with spots that have different latitudes. In such cases, $t_{s c}$ is proportional to the distance of the spot-crossing event from the center of the disk, as in the basic model
(Equation (3)). Similar considerations show that here we also get, as in Equation (12),

$$
\mathrm{TTV}_{\mathrm{sc}} \simeq-(1-\alpha) \mathcal{R} \frac{\Delta_{\mathrm{tr}}}{2} \cos \psi_{\mathrm{sc}} \sin \psi_{\mathrm{sc}},
$$

which is true for small and large spot cases alike. The extension for limb darkening also holds in this case.

\subsection{Comparing the Different TTV Patterns}

To visualize the expected TTVs derived by our analytical approximation for non-vanishing impact parameter cases, we plotted in Figure 4 the calculated TTVs for different values of the impact parameter, with the large-spot approximation, using $R_{\text {spot }} / R_{*}=0.15$ and $R_{\mathrm{pl}} / R_{*}=0.05$ values. We chose a typical parameter for a transiting system-a planet orbiting a star with solar radius in a three-day orbit. The duration of the transit (mid-ingress to mid-egress) is about $2.62 \mathrm{hr}$, the value on which we based our estimations.

One can see in the figure that the amplitude of the induced TTV is about five minutes. The derived TTVs display almost linear slope as a function of the spot-crossing position, up to a maximum at distance of $0.6-0.85$ stellar radii from the center of the stellar disk, and then a sharp drop to zero at the edge of the stellar disk.

\section{COMPARISON WITH NUMERICAL SIMULATIONS}

As noted in the introduction, Boisse et al. (2012) and Oshagh et al. (2013a) developed a numerical tool, SOAP-T, ${ }^{7}$ to simulate stellar photometric modulations induced by a rotating spot, including a planetary transit light curve which includes a spot-crossing event. Oshagh et al. (2013b) used SOAP-T to derive detailed transit light curves, and then fitted them with transit templates to obtain the expected TTVs, very similar to what is performed when deriving the TTVs from the Kepler actual data (e.g., Mazeh et al. 2013). This is a much more accurate derivation than that of the previous section, where we estimated the TTVs by the center-of-light approach. It is therefore useful to compare the TTVs obtained by our analytical approximation with the ones derived with the SOAP$\mathrm{T}$ numerical code and the transit fitting.

To do that we perform in this section two comparisons. First, we ourselves used the publicly available SOAP-T tool to produce transit light curves with spot-crossing events and fitted them with the Mazeh et al. (2013) codes to produce TTVs for a few cases and compare them with the analytical approximations. Second, we derive with our analytical center-of-light approach some TTVs for the cases derived by Oshagh et al. (2013b), and compare the results.

In Figure 5 we plotted our analytical approximation for the same system as before-a three-day transiting planet orbiting a solar-like star. We used limb darkening of $g_{1}=0.29$ and $g_{2}=0.34, R_{\mathrm{pl}} / R_{*}=0.1$ and $R_{\text {spot }} / R_{*}=0.1$, a dark spot, with $\alpha=0$, and impact parameter of zero. We can see from the figure that the maximum expected TTV based on our approximation is similar to the one obtained when simulating the spot-crossing event. The obvious difference is the phase dependence - while the analytical approximation has a smooth rise to the maximum, at phase of 0.65 , the simulated light

http://www.astro.up.pt/resources/soap-t/ 


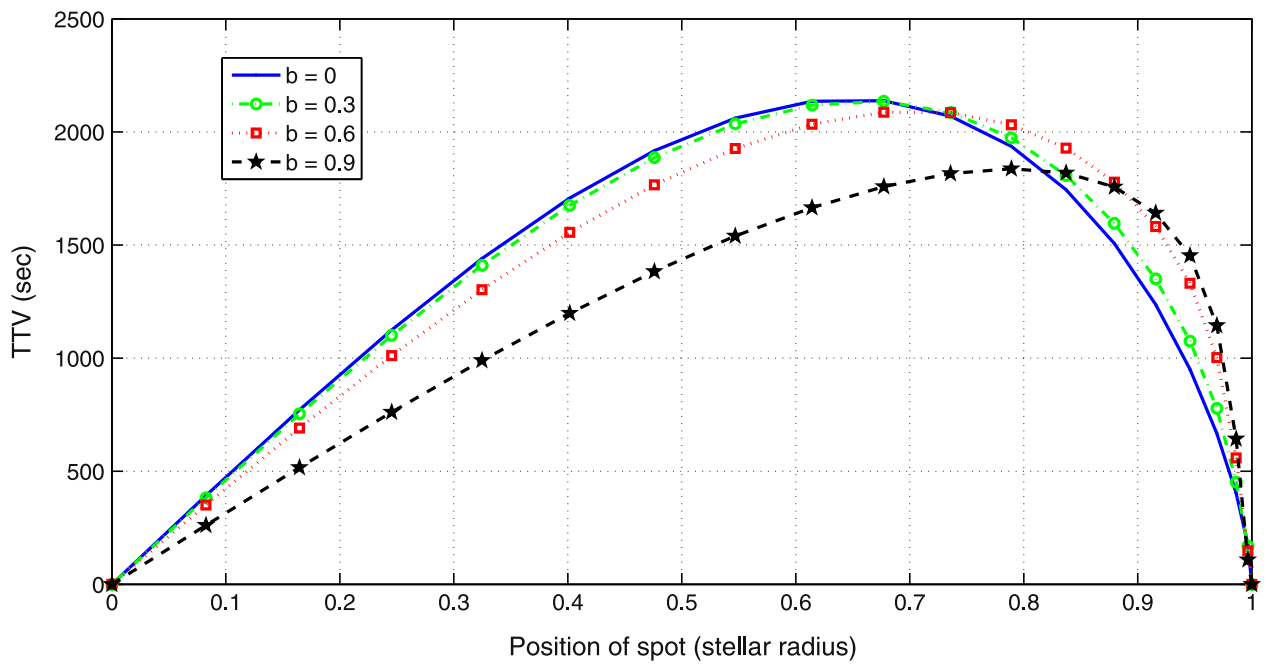

Figure 4. Analytic approximation for the induced TTV as a function of the spot-crossing position on the stellar disk for different values of the impact parameter, using the large-spot expression of Equation (22). The position of the spot-crossing event is measured relative to the center of the stellar disk, in units of the stellar radius. The graphs are for a Jupiter-size-planet that orbits a star with solar radius in a three-day orbit. The duration of the transit (mid-ingress to mid-egress) is about $2.62 \mathrm{hr}$, the value on which we based our estimations. The spot and planet radii were chosen as $R_{\text {spot }} / R_{*}=0.15$ and $R_{\mathrm{pl}} / R_{*}=0.05$. The limb darkening coefficients used are $\left[g_{1}, g_{2}\right]=[0.29,0.34]$.

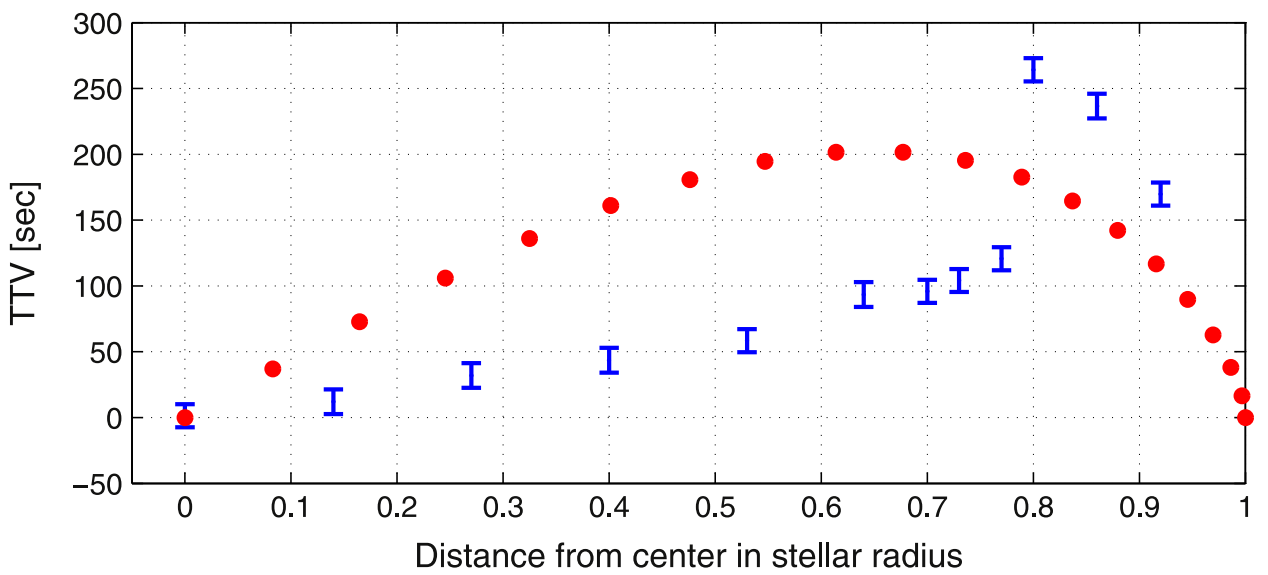

Figure 5. Comparison of the analytic approximation for the induced TTV with numerical simulations, as a function of the spot-crossing phase. The approximated TTV (red) was derived by Equation (14), while the light curves obtained by the SOAP-T tool (blue) were analyzed to derive the TTV. The error bars were derived from the Mazeh et al. (2013) codes. $R_{\mathrm{pl}} / R_{*}=0.1$ and $R_{\text {spot }} / R_{*}=0.1$. The limb darkening coefficients used were $\left[g_{1}, g_{2}\right]=[0.29,0.34]$.

curves yielded TTVs that are quite small for most phases, and rise sharply toward the maximum at phase 0.8 .

The reason for this difference comes from the different approaches of obtaining the TTV. The approach that fits a model to the simulated light curve sometimes ignores the "bump" in the light curve caused by the spot-crossing event, yielding a small TTV, while the center-of-light model is, in fact, integrating over the whole transit light curve. We will see this difference again and again. Nevertheless, this difference does not change the result of this paper-the negative (positive) correlation for prograde (retrograde) motion, as will be shown below.

Oshagh et al. (2013b) paper includes two figures that present their derived TTVs as a function of the orbital phase of the spot-crossing event. We applied our analytical approximation to all cases included in the Oshagh et al. (2013b) figures, presented in the next two figures. In Figure 6 we plot results of our analytical approximation that correspond to the six cases of Oshagh et al. (2013b, their
Figure 3), where they have considered different spot and planets relative sizes, keeping the same limb darkening parameters. We chose the same $\left(R_{\text {spot }} / R_{*}\right)^{2}$ (what they call " $f$ ") values- 0.01 and 0.0025 , and the same $R_{\mathrm{pl}} / R_{*}$ values$0.05,0.1$, and 0.15 . We used the same limb darkening coefficients of $\left[g_{1}, g_{2}\right]=\left[\begin{array}{lll}0.29, & 0.34\end{array}\right]$, and assumed a completely dark spot $(\alpha=0$ in our notation). As before, the transit duration is set to be $2.62 \mathrm{hr}$.

As in the previous figure, we see here that the maximum TTV is similar to the values obtained by Oshagh et al. (2013b), while the phase behavior of the two approaches is different, as explained above.

Another comparison was done by constructing Figure 7 and comparing it with Figure 6 of Oshagh et al. (2013b) to study the effect of limb darkening and spot darkness. Here again the amplitudes of the analytical approximation are similar to those of Oshagh et al. (2013b), while the phase dependence is different, as in our Figure 6. 


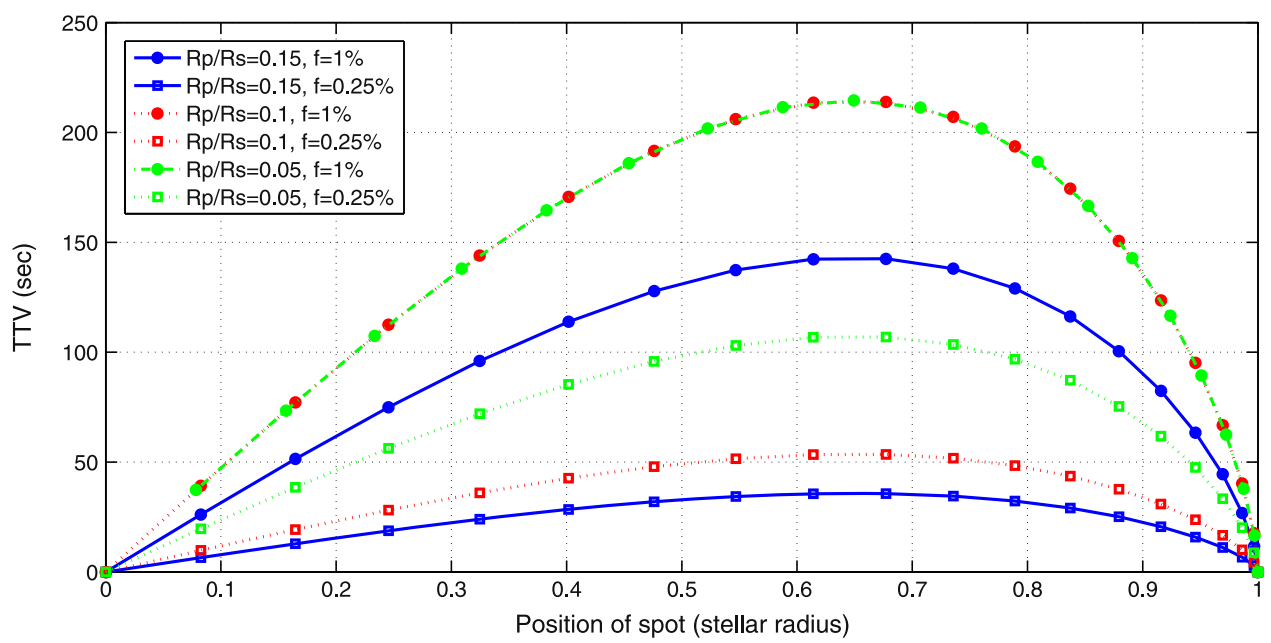

Figure 6. Analytic approximation for the induced TTV as a function of the spot-crossing phase for different spot and planet sizes. Expected TTVs were derived using Equation (14). $R p / R s$ is planet to star radius ratio and $f$ is spot to star radius ratio squared. The limb darkening coefficients used are $\left[g_{1}, g_{2}\right]=[0.29,0.34]$.

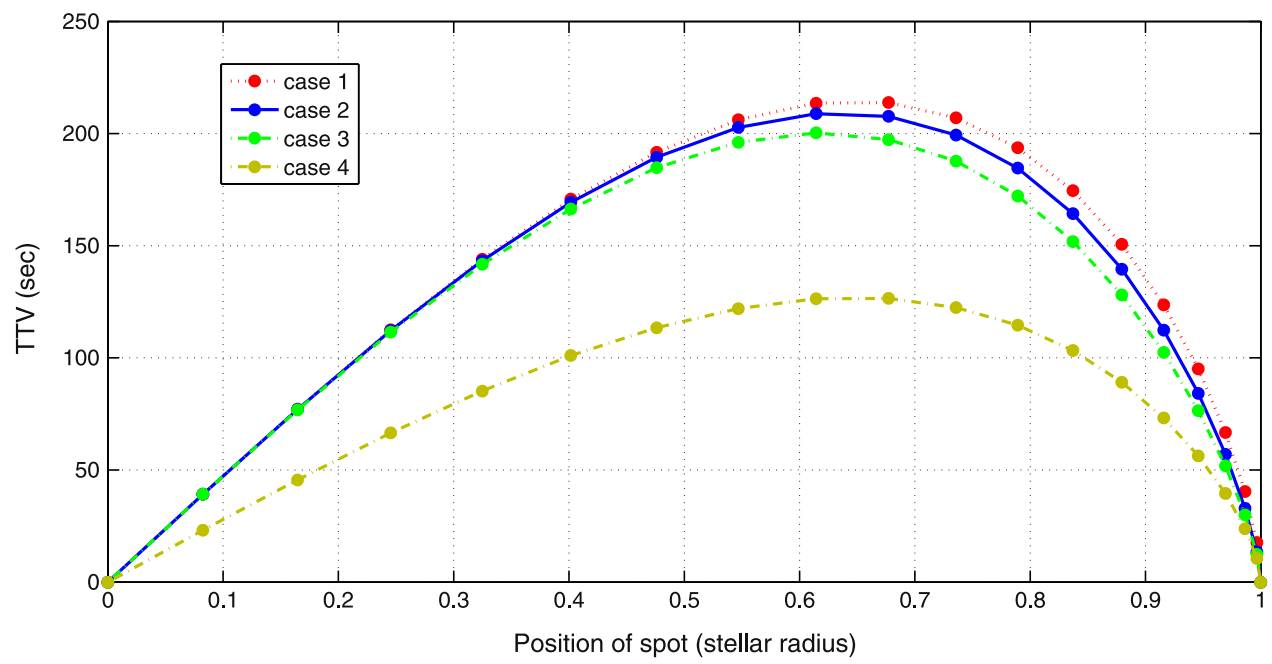

Figure 7. Expected TTVs for different limb darkening parameters, using our analytical approximation for $R_{\mathrm{pl}} / R_{*}=R_{\mathrm{spot}} / R_{*}=0.1$. The limb darkening coefficients were in case $1\left[g_{1}, g_{2}\right]=[0.29,0.34]$, in case $2[0.38,0.37]$, in case $3[0.6,0.16]$, and in case $4[0.29,0.34]$. In case 4 the spot has half of the stellar brightness $(\alpha=0.5)$ , and the spot size increased by 1.4, in order to get similar amplitude of the TTVs.

\section{ANALYTICAL APPROXIMATION FOR THE STELLAR PHOTOMETRIC SLOPES}

We turn now to approximate the local photometric slope at the time of the transit, assuming as before that the stellar brightness is modulated by a single circular spot.

For no limb darkening and null impact parameter we approximate the stellar flux, modulated by the spot as

$$
F_{*}(t) \simeq 1-\mathcal{A} \cos \psi(t), \quad \text { for }-\pi / 2 \leqslant \psi \leqslant \pi / 2,
$$

where $\mathcal{A}$ is the observed amplitude of the photometric modulation. This is so because the spot area on the stellar disk is reduced by the aspect ratio $\cos \psi$. The derivative of the stellar photometric brightness is therefore

$$
\dot{F}_{*}(t) \simeq \omega_{*} \mathcal{A} \sin \psi(t) .
$$

The amplitude of the observed stellar photometric modulation is a function of the spot radius and darkness. To express this relation we introduce the $0<\beta<1$ parameter, which accounts for the possibility that the spot crossed by the planet might not be the only spot that contributes to the stellar modulation with the observed phase. Therefore, $\beta$ measures the ratio of the area of the spot being crossed by the planet to the total neighboring spotted area that causes the photometric modulation with the same phase. The total stellar modulation due to the spots, relative to the maximum stellar brightness, is

$$
\mathcal{A} \simeq \frac{1-\alpha}{\beta}\left(\frac{R_{\mathrm{spot}}}{R_{*}}\right)^{2}
$$

In the case of limb darkening, the brightness of the spotted star takes the form

$$
\begin{aligned}
F_{*}(t) \simeq 1 & -\mathcal{A} \cos \psi(t)\left\{1-g_{1}(1-\cos \psi(t))\right. \\
& \left.-g_{2}(1-\cos \psi(t))^{2}\right\},
\end{aligned}
$$

as the photometry is modulated by the aspect ratio and the limb darkening at the spot's location. The photometric derivative is 


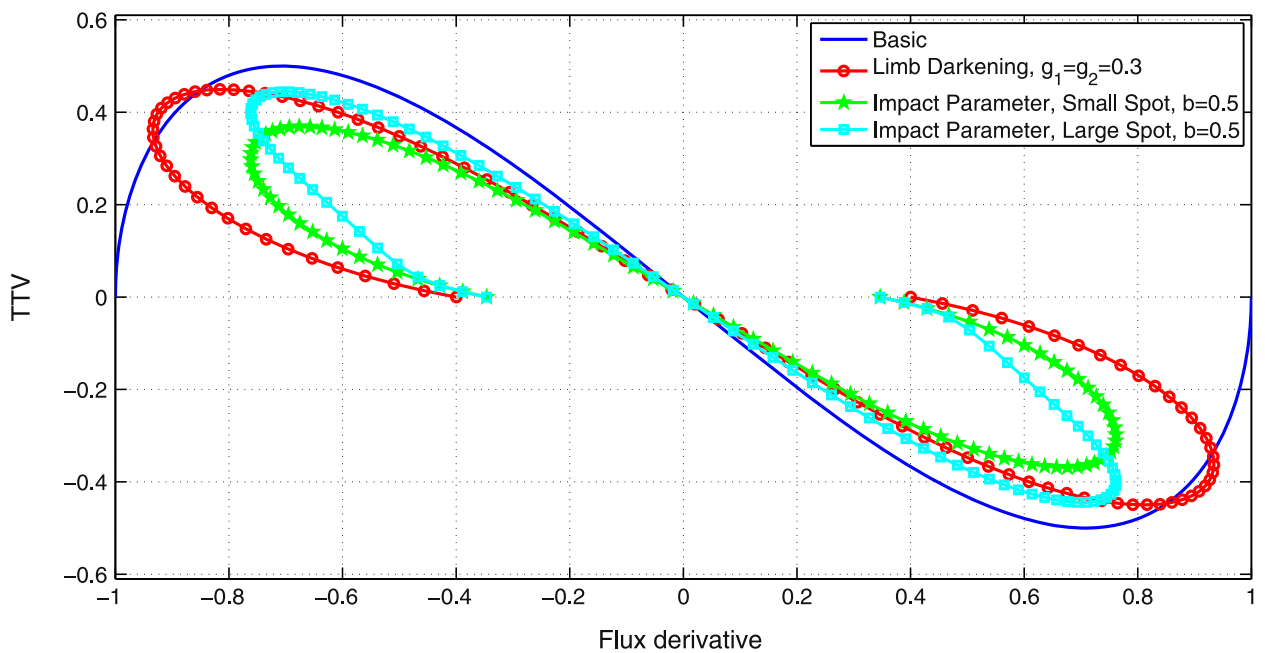

Figure 8. Induced $\mathrm{TTV}_{s c}$ versus the photometric slope for prograde motion, using arbitrary units on both axes. The blue line is the basic model, for $b=0$ and no limb darkening. The red line presents the limb-darkening, $g_{1}=g_{2}=0.3$, model, the green one is for $b=0.5$ and small spot, and the cyan line is for the same $b$ with the large spot approximation.

then:

$$
\begin{aligned}
\dot{F}_{*}(t)= & \mathcal{A} \omega_{*}\left\{\left(1-g_{1}-g_{2}\right) \sin \psi(t)\right. \\
& +\left(2 g_{1}+4 g_{2}\right) \sin \psi(t) \cos \psi(t) \\
& \left.-3 g_{2} \sin \psi(t) \cos ^{2} \psi(t)\right\} .
\end{aligned}
$$

The stellar photometry for the non-vanishing impact parameter is expressed as in Equation (23), but now $\cos \psi(t)=\sin \theta \cos \omega_{*} t$, and therefore the stellar photometry derivative is

$$
\dot{F}_{*}(t) \simeq \omega_{*} \mathcal{A} \sin \theta \sin \phi(t)
$$

where $t$ is the time since the spot was in the middle of its trail, on the projection of the stellar spin (see below) on the stellar disk, and $\omega_{*}$ is the stellar rotation rate, as explained in Section 3.3.4.

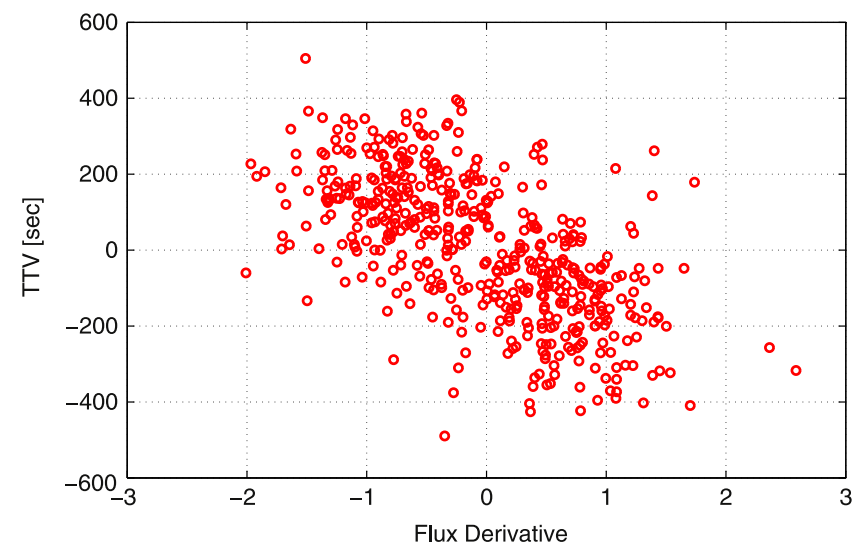

Figure 9. Simulation of TTVs, derived by the analytical approximation, versus the corresponding photometric slope for prograde motion, both with added normally distributed random noise. The noise rms equals to $50 \%$ of the maximum of the corresponding variable. The slope is scaled such that its maximum (before adding the noise) is unity. The simulation includes 500 phases selected at random. Correlation is -0.62 . See text for details.
For the non-vanishing impact parameter and stellar limb darkening the stellar photometry is

$$
\begin{aligned}
F_{*}(t) \simeq & 1-\mathcal{A} \sin \theta \cos \phi(t)\left\{1-g_{1}(1-\sin \theta \cos \phi(t))\right. \\
& \left.-g_{2}(1-\sin \theta \cos \phi(t))^{2}\right\}
\end{aligned}
$$

and its derivative is

$$
\begin{aligned}
\dot{F}_{*}(t) \simeq & \omega_{*} \mathcal{A}\left\{\left(1-g_{1}-g_{2}\right) \sin \theta \sin \phi(t)\right. \\
& +\left(2 g_{1}+4 g_{2}\right) \sin ^{2} \theta \sin \phi(t) \cos \phi(t) \\
& \left.-3 g_{2} \sin ^{3} \theta \sin \phi(t) \cos ^{2} \phi(t)\right\} .
\end{aligned}
$$

When the obliquity of the system is non-vanishing, the spot moves on a chord orthogonal to the projection of the stellar rotational axis, at a colatitude $\theta_{\text {spot }}$, with $b_{\text {spot }}=\cos \theta_{\text {spot }}$. The spot chord is different from that of the planet, which we assume goes through the center of the stellar disk. Because of the inclination of the transit chord, at the time of crossing

$$
\sin \theta_{\text {spot }} \sin \omega_{*} t=\sin \psi_{\mathrm{sc}} \cos \eta
$$

where $t$ is the time since the spot was in the middle of its trail, on the projection of the stellar spin on the stellar disk, and $\omega_{*}$ is the stellar rotation rate. Therefore, the stellar photometric derivative is like Equations (28) or (30), except for a $\sin \theta_{\text {spot }}$ factor. Note that when $\eta \rightarrow \pi / 2$ then $\dot{F}_{*}(t) \rightarrow 0$, because the spot-crossing effect occurs near the photometric maximum, and therefore the correlation with the TTVs becomes difficult to detect.

\section{THE CORRELATION BETWEEN $T T V_{s c}$ AND THE STELLAR PHOTOMETRIC SLOPES}

We are now ready to consider the expected correlation between the TTVs induced by the spot-crossing events and the local slope of the stellar photometry at the time of the transit.

\subsection{TTV as a Function of the Photometric Slope}

Figure 8 displays our analytical approximation for the TTVs as a function of the photometric derivatives for a few cases. 


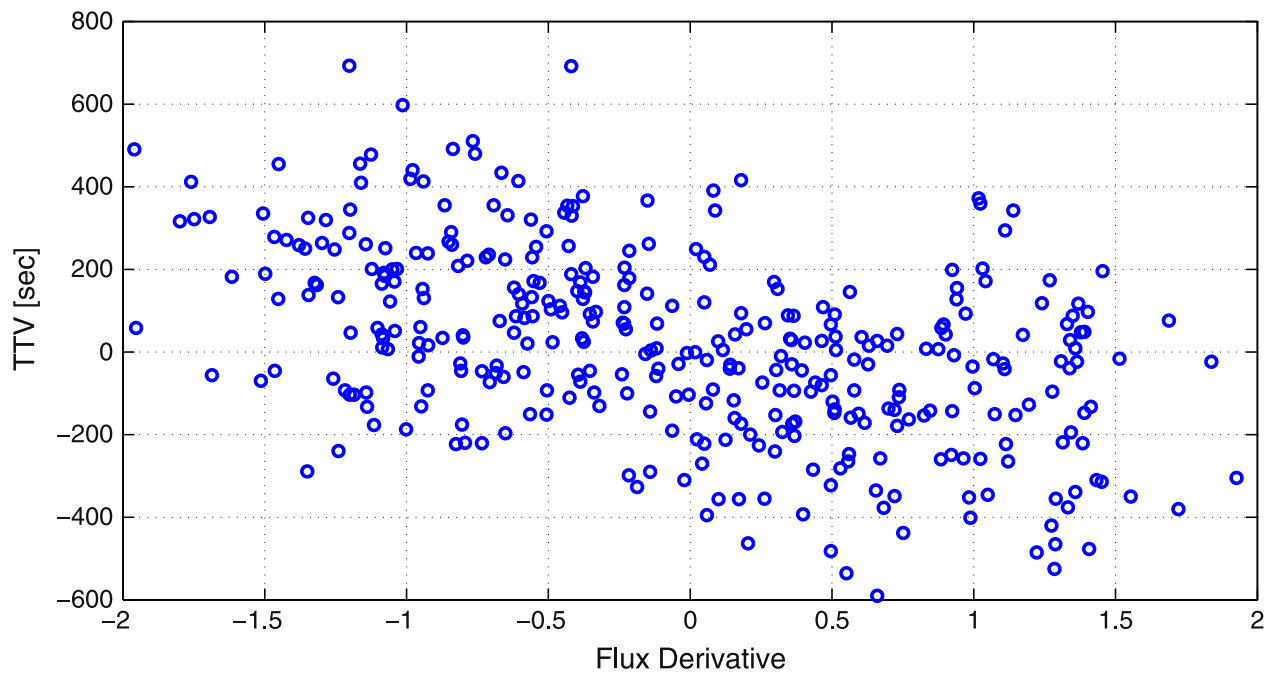

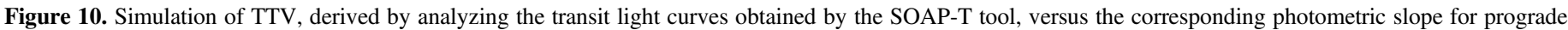
motion, both with added normally distributed random noise. Correlation is -0.48 . See Figure 9 and text for details.

The figure shows that the slope of the stellar brightness at the time of each transit and the corresponding induced TTV have opposite signs for prograde motion, and therefore we expect negative correlation between the two. Obviously, the slope and the induced TTV have the same sign for retrograde motion, because the argument presented in Section 2 and plotted in Figures 1 and 2 still holds, and therefore a positive correlation is expected in such a case.

\subsection{Correlation as a Function of Noise and Number of Observed Transits}

Figure 8 portraits how the TTVs derived by our analytical approximation depend on the photometric slope, but it does not show the real expected TTV, nor includes any observational noise, associated with every derived TTV and photometric derivative series. To see how these two affect the expected correlation we added normally distributed noise to both the simulated TTVs and the photometric derivatives, the results of which are plotted in Figure 9 for our analytically approximated TTVs, and in Figure 10 for TTVs derived by the SOAP-T tool.

In both figures we used the same fiducial system, but now with $R_{\text {spot }} / R_{*}=R_{\mathrm{pl}} / R_{*}=0.1$ and $\left[g_{1}, g_{2}\right]=[0.29,0.34]$. The photometric derivative was scaled so that its maximum was unity. We chose at random 500 phases to be plotted in the figures, and added randomly distributed normal noise to both the TTVs and the slope derivatives. The noise rms was equal to $50 \%$ of the maximum of the corresponding variable. This amounts to a $150 \mathrm{~s}$ error on the TTVs and 0.5 to the scaled slope.

The two figures show similar results-there is a very clear anti-correlation between the induced TTVs and the photometric slopes at the transit timings, even when some small noise is added. In fact, the noise covers up the fact that for some phases the dependence of the TTVs on the slope changes its sign, as we see in Figure 8.

To estimate the expected effect of the noise on the measured correlation we ran extensive simulations, with different values of noise level and number of observed transits. For each choice of noise level, $\sigma_{\mathrm{TTV}}, \sigma_{\text {slope }}$, and number of transits, $N$, we chose $N$ random phases, derived their TTVs and photometric derivatives, added randomly distributed noise to both the
TTVs and the stellar photometric slopes, and then derived the resulting (anti-)correlation. We repeated this simulation 1000 times, with the same values of noise level and number of points. We then derived the median and scatter of the sample of correlations obtained, which are plotted in Figure 11 as a function of the noise level and $N$.

We chose five values for $\sigma_{\mathrm{TTV}}$ and $\sigma_{\text {slope }}$, each scaled as a fraction of the maximum of its corresponding variable. The five signal-to-noise ratios we chose were $[0,0.15,0.3,0.5,1]$. Each choice characterizes both the noise added to the TTVs and to the photometric slopes. For $N$ we chose values of 50, 100, 500, and 1000. For short-period transiting planets Kepler light curves could have on the order of 1000 transits, but 200-400 was a more typical number. All together we had $4 \times 5=20$ sets of simulations, the results of which are plotted in Figure 11.

The expected value of the correlation depends on the noise level. It goes from 0.9 for no noise down to 0.3 for a signal-tonoise ratio $(\mathrm{S} / \mathrm{N})$ of unity. The $1 \sigma$ spread of the correlation depends on the noise level and the number of points. It goes from 0.13 for $N=50$ and $\mathrm{S} / \mathrm{N}$ of unity down to 0.02 for $N=1000$ and no noise. The figure suggests that we can easily detect the correlation with $\mathrm{S} / \mathrm{N}$ of unity, if we can measure on the order of 500 TTVs and their corresponding photometric slopes.

\section{DISCUSSION}

We presented here a simple approach that can, in a few cases, use the derived TTVs of a transiting planet to distinguish between prograde and retrograde planetary motion with respect to the stellar rotation, assuming the TTVs are induced by spotcrossing events. Using a simplistic analytical approximation we showed that those TTVs might have negative (positive) correlation with the local stellar photometric slopes at the transit timings for prograde (retrograde) motion. We have shown that the correlation might be detected for different stellar limb darkening and different impact parameters. Furthermore, we obtained similar correlated TTVs when we used the SOAP$\mathrm{T}$ tool to simulate transit light curves and derive the corresponding TTVs. We have shown also that even if we include a certain amount of noise, the correlation is still detectable. 


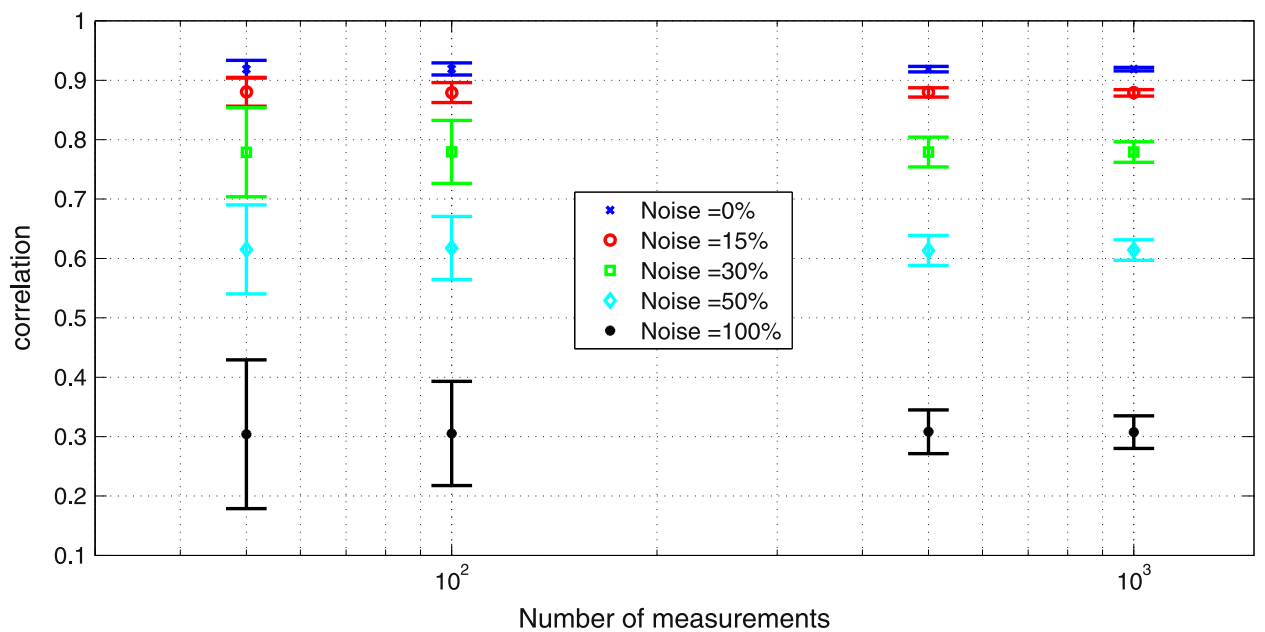

Figure 11. Absolute value of the correlation of 1000 system samples of simulated induced TTVs with the stellar photometric slopes, for different noise levels and different number of points. The points are the median of each sample and the error bars are the sample rms. See text for details.

Can such a correlation surface above the observational noise? The expected amplitude of the TTV can be estimated by Equations (11) and (13). For example, a system with $R_{\text {spot }} \simeq R_{\mathrm{pl}} \simeq 0.1 R_{*},(1-\alpha) / 4 \simeq 0.25$, and transit duration of $3 \mathrm{hr}$ should show an induced TTV on the order of five minutes. So, we can expect to observe the (anti-) correlation between the TTVs and the photometric slopes only for systems with high enough $\mathrm{S} / \mathrm{N}$ that allows timing precision of the order of five minutes or better. Note that for a three-day transiting planet in the Kepler field we have at hand data for up to about 400 transits, enabling us to detect a correlation even if the noise is comparable with the signal.

Obviously, the approximation and simulation presented here are quite simplistic. First, spotted stars probably have more than one spot. The spot eclipsed by the planet might not be the one dominating the stellar flux modulation, and hence the local photometric slope at the time of transit might be very different from the expressions we developed here. Note, however, that in our simulation we allowed an error of the photometric slope that can be as large as the slope itself, and showed that even in such a case the correlation still can be detected. Second, spots have different stellar latitudes, so some transits might not have induced TTVs at all, contaminating the expected correlation. To deal with this problem one might consider the correlation of only the highly significant TTVs, which could show the signal better. Third, the system obliquity can be very different from $0^{\circ}$ or $180^{\circ}$, although most of the planets around cool stars, with a temperature below about $6000 \mathrm{~K}$, apparently are aligned with the stellar rotation (Albrecht et al. 2012; Mazeh et al. 2015). We have shown that for systems with non-vanishing obliquity and null impact parameter the shape of the dependence of the TTV on the photometric slope is the same, although the obliquity might decrease or even eliminate the correlation, because many transits might not include a spot-crossing event at all. Note, however, that even for significant obliquity the correlation might still exist, assuming there will be enough induced TTVs, probably caused by spots with different latitudes. Here again one might ignore the non-significant TTVs when searching for a correlation. Fourth, the observed transiting system might have additional planets that induce dynamical TTVs, completely shadowing the TTVs caused by spot crossing events.
Despite all these obstacles, the correlation studied here might be solid enough to show up for a few KOIs. Although our method cannot give an accurate spin-orbit angle, but can instead only indicate the sign of the orientation of the planetary motion, the method might be useful nevertheless, as it uses Kepler long-cadence data that is publicly available for all transiting planets. In the next paper (T. Holczer et al. 2015, in preparation) we report on a search for correlation between the available TTVs and the corresponding local photometric slopes at the transit timings for all Kepler KOIs, and indeed find five convincing cases with significant correlations.

The approach described here can in principle be applied to any eclipsing system, whether it is a transiting planet or a stellar binary. For a binary system, the induced observed minus calculated $(O-C)$ eclipse timings can be estimated with the small-spot approximation, for which the planetary radius is that of the secondary. We therefore expect the TTVs to be on the same order of magnitude as for transiting planets. However, as eclipses in binaries are usually deeper and longer than the planetary transits, we expect the $O-C s$ in eclipsing binaries to be more precise.

In fact, a negative correlation between the $O-C s$ and the local photometric slopes was identified already for the stellar eclipsing binary in the Kepler-47 circumbinary planet system (Orosz et al. 2012). The authors detected $O-C$ on the order of 1 minute in the timing of the primary eclipse, and used the derived linear trend to correct the eclipse timings. The detection of a negative correlation for Kepler-47 is consistent with a more detailed analysis of the spot-crossing events, also done by Orosz et al. (2012), which indicates a prograde motion.

The method presented here can be applied in the future to a large sample of systems monitored by current and future space missions, like K2 (Howell et al. 2014), TESS (Ricker et al. 2014), and PLATO (Rauer et al. 2014), helping discovering, without additional observations, interesting systems that are worth following, and possibly find what are the conditions for alignment or misalignment of stellar rotations and orbital motions of planets and stellar binaries.

We are grateful to the referee for very helpful comments that helped us substantially improve the paper. We are thankful to the authors of the SOAP-T tool that made it publicly available. 
The research leading to these results has received funding from the European Research Council under the EU's Seventh Framework Programme (FP7/(2007-2013)/ ERC Grant Agreement No. 291352). T. M. also acknowledges support from the Israel Science Foundation (grant No. 1423/11) and the Israeli Centers of Research Excellence (I-CORE, grant No. 1829/12). T. M. is grateful to the Jesus Serra Foundation Guest Program and to Hans Deeg and Rafaelo Rebolo, that enabled his visit to the Instituto de Astrofsica de Canarias, where the last stage of this research was completed. This work was performed in part at the Jet Propulsion Laboratory, under contract with the California Institute of Technology (Caltech) funded by NASA through the Sagan Fellowship Program executed by the NASA Exoplanet Science Institute.

\section{REFERENCES}

Albrecht, S., Reffert, S., Snellen, I., Quirrenbach, A., \& Mitchell, D. S. 2007, A\&A, 474, 565

Albrecht, S., Reffert, S., Snellen, I. A. G., \& Winn, J. N. 2009, Natur, 461, 373

Albrecht, S., Winn, J. N., Carter, J. A., Snellen, I. A. G., \& de Mooij, E. J. W. 2011, ApJ, 726, 68

Albrecht, S., Winn, J. N., Johnson, J. A., et al. 2012, ApJ, 757, 18

Albrecht, S., Winn, J. N., Torres, G., et al. 2014, ApJ, 785, 83

Barnes, J. W. 2009, ApJ, 705, 683

Barnes, J. W., Linscott, E., \& Shporer, A. 2011, ApJS, 197, 10

Batalha, N. M., Rowe, J. F., Bryson, S. T., et al. 2013, ApJS, 204, 24

Batygin, K. 2012, Natur, 491, 418

Boisse, I., Bonfils, X., \& Santos, N. C. 2012, A\&A, 545, A109

Chaplin, W. J., Sanchis-Ojeda, R., Campante, T. L., et al. 2013, ApJ, 766, 101

Dawson, R. I. 2014, ApJL, 790, LL31

Deming, D., Sada, P. V., Jackson, B., et al. 2011, ApJ, 740, 33

Désert, J.-M., Charbonneau, D., Demory, B.-O., et al. 2011, ApJS, 197, 14

Fabrycky, D. C., Ford, E. B., Steffen, J. H., et al. 2012, ApJ, 750, 114

Fabrycky, D., \& Tremaine, S. 2007, ApJ, 669, 1298

Gizon, L., \& Solanki, S. K. 2003, ApJ, 589, 1009

Groot, P. J. 2012, ApJ, 745, 55

Hartman, J. D., Bakos, G. Á, Noyes, R. W., et al. 2011, AJ, 141, 166
Hansen, B. M. S. 2012, ApJ, 757, 6

Hébrard, G., Ehrenreich, D., Bouchy, F., et al. 2011, A\&A, 527, L11

Hirano, T., Sanchis-Ojeda, R., Takeda, Y., et al. 2012, ApJ, 756, 66

Hirano, T., Sanchis-Ojeda, R., Takeda, Y., et al. 2014, ApJ, 783, 9

Holt, J. R. 1893, A\&A, 12, 464

Howell, S. B., Sobeck, C., Haas, M., et al. 2014, PASP, 126, 398

Irwin, J., Aigrain, S., Bouvier, J., et al. 2009, MNRAS, 392, 1456

Mazeh, T., Nachmani, G., Holczer, T., et al. 2013, ApJS, 208, 16

Mazeh, T., Peretz, H., McQuillan, A., et al. 2015, ApJ, in press (arXiv:1501.01288)

McLaughlin, D. B. 1924, ApJ, 60, 22

McQuillan, A., Mazeh, T., \& Aigrain, S. 2014, ApJS, 211, 24

Moutou, C., Díaz, R. F., Udry, S., et al. 2011, A\&A, 533, A113

Naoz, S., Farr, W. M., Lithwick, Y., Rasio, F. A., \& Teyssandier, J. 2011, Natur, 473, 187

Nutzman, P. A., Fabrycky, D. C., \& Fortney, J. J. 2011, ApJL, 740, L10

Orosz, J. A., Welsh, W. F., Carter, J. A., et al. 2012, Sci, 337, 1511

Oshagh, M., Boisse, I., Boué, G., et al. 2013a, A\&A, 549, A35

Oshagh, M., Santos, N. C., Boisse, I., et al. 2013b, A\&A, 556, A19

Queloz, D., Eggenberger, A., Mayor, M., et al. 2000, A\&A, 359, L13

Rauer, H., Catala, C., Aerts, C., et al. 2014, ExA, 38, 249

Ricker, G. R., Winn, J. N., Vanderspek, R., et al. 2014, Proc. SPIE, 9143, 20

Rossiter, R. A. 1924, ApJ, 60, 15

Sanchis-Ojeda, R., \& Winn, J. N. 2011, ApJ, 743, 61

Sanchis-Ojeda, R., Fabrycky, D. C., Winn, J. N., et al. 2012, Natur, 487, 449

Sanchis-Ojeda, R., Winn, J. N., Holman, M. J., et al. 2011, ApJ, 733, 127

Sanchis-Ojeda, R., Winn, J. N., Marcy, G. W., et al. 2013, ApJ, 775, 54

Schlaufman, K. C. 2010, ApJ, 719, 602

Schlesinger, F. 1910, PAllO, 1, 123

Shporer, A., Brown, T., Mazeh, T., \& Zucker, S. 2012, NewA, 17, 309

Slawson, R. W., Prša, A., Welsh, W. F., et al. 2011, AJ, 142, 160

Szabo, G. M., et al. 2011, ApJL, 736, L4

Szabó, R., Szabó, G. M., Dálya, G., et al. 2013, A\&A, 553, A17

Triaud, A. H. M. J., Collier Cameron, A., Queloz, D., et al. 2010, A\&A, 524, A 25

Triaud, A. H. M. J., Hebb, L., Anderson, D. R., et al. 2013, A\&A, 549, A18

Winn, J. N., Johnson, J. A., Marcy, G. W., et al. 2006, ApJL, 653, L69

Winn, J. N., Fabrycky, D., Albrecht, S., \& Johnson, J. A. 2010, ApJL, $718, \mathrm{~L} 145$

Winn, J. N., Howard, A. W., Johnson, J. A., et al. 2011, AJ, 141, 63 